\title{
Cooperation and Competition Coupled Diffusion of Multi- Feature on Multiplex Networks and its Control
}

This paper was downloaded from TechRxiv (https://www.techrxiv.org).

LICENSE

CC BY 4.0

SUBMISSION DATE / POSTED DATE

26-01-2022 / 01-02-2022

\section{CITATION}

Zhao, Dawei; Wang, Yinglong; Yang, Meihong; Li, Shudong; Wang, Zhen; Peng, Haipeng (2022): Cooperation and Competition Coupled Diffusion of Multi-Feature on Multiplex Networks and its Control. TechRxiv. Preprint. https://doi.org/10.36227/techrxiv.19069553.v1

$\mathrm{DOI}$

10.36227/techrxiv.19069553.v1 


\title{
Cooperation and Competition Coupled Diffusion of Multi-Feature on Multiplex Networks and its Control
}

\author{
Dawei Zhao, Member, IEEE, Yinglong Wang, Meihong Yang, Shudong Li, Zhen Wang, Member, IEEE, Haipeng \\ Peng
}

\begin{abstract}
Cooperation and competition widely exist in various kinds of network diffusions which however are usually studied separately. Recently, a novel network diffusion model, called multi-feature diffusion (MFD), attracts considerable attentions. The existing works usually assume that each feature diffuses independently and neglects the possible complex interplay between different features. In this paper, we introduce the cooperation and competition into the MFD and propose the cooperation and competition coupled diffusion of multi-f eature on multiplex network (CCMF). An unified framework and mathematical analytic theory regarding CCMF are then presented which are applicable and computationally efficient for any number of features and their own different sub-diffusion dynamics. In addition, an interesting finding is obtained in CCMF: compared with the high intensity competition, performing lower intensity competition under weak competition ability is more easier to result in positive effect. Due to the great importance of controlling network diffusion in many diverse contexts, we also propose an optimal allocation strategy of control resource for CCMF which first realizes the promotion and suppression of network diffusion simultaneously under one optimization framework and is also verified to be very efficient.
\end{abstract}

Index Terms-Cooperation, competition, multi-feature diffusion, multiplex network, optimization.

\section{INTRODUCTION}

Network diffusions widely exist in varies fields of nature and human society, such as the spreading of epidemic in biological network, the propagation of computer viruses in communization networks, the rumor and information diffusion in online

D. Zhao, Y. Wang and M. Yang are with the Shandong Provincial Key Laboratory of Computer Networks, Shandong Computer Science Center (National Supercomputer Center in Jinan), Qilu University of Technology (Shandong Academy of Sciences), Jinan 250014, China. (e-mail: zhaodw@sdas.org)

$\mathrm{S} . \mathrm{Li}$ is with the Cyberspace Institute of Advance Technology, Guangzhou University, Guangzhou 510006, China. (e-mail: lishudong@gzhu.edu.cn)

Z. Wang is with Center for OPTical IMagery Analysis and Learning (OPTIMAL) and School of Mechanical Engineering, Northwestern Polytechnical University, Xi'an 710072, China. (e-mail: w-zhen@nwpu.edu.cn)

H. Peng is with the Information Security Center, State Key Laboratory of Networking and Switching Technology, and National Engineering Laboratory for Disaster Backup and Recovery, Beijing University of Posts and Telecommunications, Beijing 100876, China. (e-mail: penghaipeng@bupt.edu.cn)

This work was supported by the National Key Research and Development Project of China (2020AAA0107700), the Shandong Provincial Natural Science Foundation (ZR2020YQ06), the National Natural Science Foundation of China (62072131), the Key Research and Development Project of Shandong (2019JZZY010132) and the Pilot Project for Integrated Innovation of Science, Education and Industry of Qilu University of Technology (Shandong Academy of Sciences) (2020KJC-ZD02).

Manuscript received $\mathrm{xxxx}$; revised $\mathrm{xxxx}$. social networks and the failures propagation in engineering networks [1]-[6]. They have attracted considerable attention due to their important potential applications, for example understanding the evolution mechanism of network diffusion will help to accurately predict whether a certain diffusion will outbreak or die out on its own, effectively control the undesired diffusions of disease, virus or rumor and minimize their influence, and maximize the adoption of specific product and achieve marketing goals, etc [7]-[9].

The dynamical model is the core of understanding evolution mechanism of network diffusion. The potential risk and difficulty lead to be unfeasible of studying on real network diffusion, modeling approach is therefore widely adopted to be the fundamental framework where to compare and test theories, as well as to evaluate the effectiveness of the control strategies. In the past few decades, several dynamical models, like epidemic model, independent cascading model and linear threshold model, have been proposed to characterize the evolutionary process of various kinds of network diffusions [1]. In addition, the network topology, extracted from real-world, plays a vital role on the outcomes of the diffusion process [4], [10], [11]. The diffusion on the well-known random network, small-world network and scale-free network shows significant differences of dynamic characteristic and evolutionary mechanism. For example, in networks with a power-law degree distribution with exponent $2<\gamma \leq 3$, for which $\left\langle k^{2}\right\rangle \rightarrow \infty$ in the limit of a network of infinite size, the epidemic threshold tend asymptotically to zero [12].

Prior to 2010, the majority of studies on network diffusion are mainly concentrated to the case of single diffusion process on single network. In the past decade, we have witnessed a tremendous shift that the focus of complex networks was extended from single network to multiplex network [13]. The multiplex network is coupled by several network layers, each of which has same network nodes but different topologies and dynamics. Thus far, the topological characteristics of multiplex networks and dynamical process (such as evolutionary game, diffusion and synchronization) upon them have attracted great attention in both theoretical and empirical areas [14]-[17]. As a consequence, several network diffusion dynamics on multiplex network were proposed, such as the multiple route diffusion (MRD) and multiple information diffusion (MID) [18]-[20]. The MRD characterizes the phenomenon that one event (for example one piece of information, one epidemic, 
one computer virus, etc) diffuses through multiple routes simultaneously. While the MID is for multiple event and each diffusion is for one event.

Most recently, a novel diffusion model on multiplex network was introduced, i.e. the multi-feature diffusion (MFD), which is for one event with multiple features [21], [22]. In MFD, the outcomes of the event is determined by the diffusion of the multiple features. In addition, the features could be subdivided into positive and negative categories. The MFD matches to a lot of realistic scenario. For example, one produce usually has its own multiple feature, including the price, appearance, function, quality and so on. Whether the customer will buy the produce or not depends on his/her overall evaluation of all these features [21]. As to one topic in social network, it may contains multiple related messages, both positive and negative. The users accept or deny the topic depending on the comprehensive consideration of all related messages [22].

Research on multi-feature diffusion is still in its infancy, several challenges remain. The number of features in MFD maybe large and different feature may diffuses following its own different dynamical model. In addition, the features can be impossible to diffuse independently since they all around one same event. The interplay between features is also pluralistical rather than monistical, which may include both cooperative relationship between same type of features and competitive relationship between different type of features. The unified framework and mathematical analytic theory regarding MFD with the cooperation and competition interplay between features (CCMF, cooperation and competition coupled diffusion of multi-feature) should be developed.

One of the main purposes of studying network diffusion is to recognize and understand its dynamic characteristic and evolutionary mechanism, and then control it efficiently [7], [23]. Two important problems regarding the control of network diffusion are the optimal selection of node sets either to minimize or maximize the extent of outbreaks under the constraint of limited resources [24]-[27]. The minimization problem is equivalent to the network immunization problem which aims to minimize the outbreak size of the diffusion by immunizing a node set of "superblocks" [24], [25]. While the maximization problem is to identify a set of "superspreaders" which are chosen as the initiators of the diffusion, the outbreak size will be maximized [26], [27]. The identification of these "supernodes" is often done by employing the centrality index of the underlying network topology, such as betweenness, degree, pagerank, eigenvector, closeness, k-shell and Collective Influence etc [28], [29]. Another type of solution depends not just on the network topology, but also on the specific dynamics of the diffusion, including the Monte-Carlo (MC) methods, integer/linear programming, greedy algorithms and the message-passing algorithm [26], [30], [31].

Obviously, the minimization problem and the maximization problem of control belong to two opposite problems and thus usually would not be appear in the same application scenarios. Similarly, one of the main control goals of CCMF is also to minimize or maximize the outcomes of the event. However, the realization of control CCMF covers the minimization problem and maximization problem simultaneously due to the coexistence of negative features and positive features around the event. Specifically, the maximization (minimization) of CCMF could be realized through the minimization (maximization) of negative features and the maximization (minimization) of positive features simultaneously. The efficient theory and method regarding such control issue are absent.

In this paper, we focus on the cooperation and competition coupled diffusion of multi-feature on multiplex networks and its control. Our contributions are summarized as follows:

- We introduce the cooperation and competition into the MFD and propose the cooperation and competition coupled diffusion of multi-feature on multiplex network (C$\mathrm{CMF}$ ). An unified framework and mathematical analytic theory regarding CCMF are then presented which are applicable and computationally efficient for any number of features and their own different sub-diffusion dynamics.

- An interesting finding is obtained in CCMF: compared with the high intensity competition, performing lower intensity competition under weak competition ability is more easier to result in positive effect.

- We propose an optimal allocation strategy of control resource for CCMF which first realizes the promotion and suppression of network diffusion simultaneously under one optimization framework and is also verified to be very efficient.

The remainder of this paper is organized as follows: Section II surveys existing works on network diffusion and its control problem. Section III proposes the cooperation and competition coupled diffusion of multi-feature on multiplex network and introduces the unified framework and mathematical analytic theory. Section IV presents the optimal allocation strategy of control resource for CCMF. Section V performs model analysis and verifies the efficiency of the optimization algorithm. Conclusion is shown in Section VI.

\section{Related Work}

The study of network diffusion origins from multiple scientific fields, such as health science, information science, social science and marketing science which involve the diffusions of epidemic, information, social behaviors and innovation respectively [1], [5], [32]. These studies resulted in various kinds of diffusion models that help to analyze and predict the dynamical characteristics from the outbreak to process behavior to the stable state of the diffusion. The existing diffusion models can be broadly divided into two levels, the macro- and micro-level. The Bass model is one of the well known macro-model whose most important character is that each individual is assumed to be related to every other individuals, i.e., no network knowledge is needed [33]. The micro-model, in contrast, requires the complete knowledge of the network node-node relationships, and the knowledge of influence probability between any two neighboring nodes. Examples of these models include epidemic model (which can 
be subdivided into the SI model, SIS model, SIR model, etc.), independent cascading model and linear threshold model [1]. In this sense, the network structures play vital role on the dynamical characteristics of network diffusion, including the macro-, meso- and micro-level. For example, form the macrolevel, Pastor-Satorras and Vespignani found the absence of epidemic threshold and critical behavior in a wide range of scale-free network, which indicated the infections can proliferate on scale-free networks whatever spreading rates they may have [12]. At the meso-level, Ref. [34] found that networks with strong community structure are helpful for reducing the danger brought by epidemic prevalence. In addition, at the micro-level, it's known that the hub nodes are better able to promote and suppress the network diffusion [28].

From 2010, the research focus of network diffusion was changed from the single diffusion on top of single network to the multiple diffusion on multiplex network, such as the multiple route diffusion (MRD) and multiple information diffusion (MID) [13], [18]-[20], [35]. The MRD and MID introduced novel complex dynamical behaviours, the most typical one of which is the interplay between different sub-diffusions. Granell et al. presented the analysis of the interrelation between two processes accounting for the spreading of an epidemic, and the information awareness to prevent its infection, on top of multiplex networks [20]. Wang et al. investigated the coevolution mechanisms and dynamics between information and disease diffusions and found that there is an optimal information transmission rate that markedly suppresses the disease diffusion [36]. Zhao et al. developed an efficient patch distribution scheme by using the competition mechanism between patch dissemination and virus propagation [37]. Wang et al. explored the influence of positive and negative preventive information on epidemic propagation and found that the epidemic prevalence and the epidemic outbreaks can be suppressed by the diffusion of positive preventive information and be promoted by the diffusion of negative preventive information [38]. Wei et al. investigated the cooperative diffusion, wherein the virus spreading in one layer can penetrate into the other to promote the diffusion process and found the global diffusion threshold of is smaller than the epidemic thresholds of the corresponding isolated networks [39]. Wei et al. proposed a framework can unify three scenarios for various mutual influences between the two diffusion processes, including competing diffusion, cooperative diffusion and the combination of the two [40].

In 2019 , Li et al. introduced a novel diffusion dynamics of multiple social networking messages associated with the same topic and the cooperative relationship and competitive relationship between messages were also considered [41]. However, the proposed analysis framework lacks extensibility in face of the diffusions of a large number of messages. For the same issue, $\mathrm{Wu}$ et al. presented the formal definition, called Multi-Feature diffusion (MFD) [21], [22]. They also discussed the optimal control issues of the MFD in two application scenarios, including the diffusion maximization of product and the minimization of social rumor. But they assumed that the features diffused independently and ignored the possible interplay between the features, such as the cooperative relationship and competitive relationship.

The identification of "supernodes" in control can be divided into two categories, structural method and functional method [28], [42]. The former purely depends on the network topology, especially the centrality index including the degree, between, K-shell, CI. The graph-partitioning, explosive percolation and belief-propagation guided decimation (BPD) algorithm [30], [43], [44] are also good structural methods. Ribalta et.al. extended centrality index to multiplex network and proposed the betweenness, rigenvector, pagerank and clustering coefficients of multiplex network [45]-[47]. Osat et al. reframed the collective influence, explosive immunization and simulated annealing to identify the supernodes of multiplex network [48]. Due to the ignorance of dynamic characteristics of network diffusion, the structural method is not always effective and may have quite different performance under different network and diffusion process. The functional method depends not just on the topology, but also on the specific dynamics considered, thus be generally more effective. Altarelli et al. proposed message-passing-based algorithm to identify the optimal immunization nodes for SIR model and SIS model [49]. The discrete particle swarm optimization technique was developed to optimize the local influence criterion for the independent cascade model [50]. Chen et al. combined the perturbation theory with Lagrange multiplier method to derive the optimal allocation of the curing rates to suppress the epidemic spreading [31]. Lokhov and Saad used the dynamic message-passing approach for spreading processes and introduced an efficient, versatile and principled optimization framework for solving dynamic control resource allocation problems in spreading processes [26]. In addition, Wang et al. designed an extended influence spreading model, based on which a memetic algorithm was developed to find the influential nodes of multiplex networks [29]. However, these existing works on controlling diffusion mainly studied the promotion and suppression of diffusions separately.

\section{COOPERATION AND COMPETITION COUPLED DIFFUSION OF MULTI-FEATURE ON MULTIPLEX NETWORK}

In this section, we introduce the cooperation and competition into the MFD and propose the novel model of CCMF. An unified framework and mathematical analytic theory regarding CCMF are then presented.

Considering an event $\mathbb{E}$ contains $m$ features $\{A, B, C \ldots\}_{m}=$ $\Delta_{\mathbb{E}}^{+} \cup \Delta_{\mathbb{E}}^{-}=\Delta_{\mathbb{E}}$, where $\Delta_{\mathbb{E}}^{+}$and $\Delta_{\mathbb{E}}^{-}$represent the sets of positive features and negative features of $\mathbb{E}$ respectively, and $\Delta_{\mathbb{E}}^{+} \cap \Delta_{\mathbb{E}}^{-}=$ $\emptyset$. The different features may diffuse through different relationships betweem network nodes. Therefore, the underlying network framework of the MFD should be a multiplex network formed by $m$ different layers, i.e. $G=\left\{G^{A}, G^{B}, G^{C} \ldots\right\}_{m}$, each of which corresponds to one feature. Every layer $G^{A}=\left(V, E^{A}\right)$ has a same set $V=\{\ldots, i, j, \ldots\}_{N}$ of $N$ nodes and a distinct set of edges $E^{A}=V \times V$.

In MFD, different feature may have their own different dynamical behavior, therefore may follow different sub- 
diffusion dynamical model. For simplicity, we utilize the same Susceptible-Infected-Recovered (SIR) model to characterize the diffusions of all features. But the following presented framework and methods are directly applicable to many different diffusion dynamical model. Specifically, in the SIR model the nodes in a network layer, e.g. $G^{A}$, can be in one of the three states: $A$-susceptible $\left(S_{A}\right)$ state in which the node is free of the feature $A$ but can be infected via contacts with $A$-infected neighbors; $A$-infected $\left(I_{A}\right)$ state, where the node accepted the feature $A$ and can transmit it to its $S_{A}$ neighbors; and $A$-recovered $\left(R_{A}\right)$ state, in which the node still accepted the feature $A$ but has no willing to pass $A$ to other nodes. We use the discrete time step for the evolution of SIR process and at each time step $t$, the $I_{A}$ node $j$ can infect all its $S_{A}$ neighbor $i$ with transmissibility $\phi_{j i}^{A}(t)$, and then becomes $R_{A}$ node with probability $\varphi_{j}^{A}(t)$.

Let $p_{i}^{S_{A}}(t), p_{i}^{I_{A}}(t), p_{i}^{R_{A}}(t)$ be the probabilities of node $i$ be in $S_{A}$ state, $I_{A}$ state, $R_{A}$ state at time step $t$, respectively. Here we utilize a novel way to represent the transmissibility based on the sigmoid function

$$
\phi_{j i}^{A}(t)=\frac{1}{1+e^{-\left(\beta_{j i}^{A}(t)+\alpha^{A} r_{i}^{A}(t)\right)}}
$$

where

$$
r_{i}^{A}(t)=\sum_{B \in \Delta_{\mathbb{E}}, B \neq A}\left(p_{i}^{I_{B}}(t)+p_{i}^{R_{B}}(t)\right) h^{B A} .
$$

$\alpha^{A}$ is a tunable parameter and $\alpha^{A}>0 . p_{i}^{I_{B}}(t)+p_{i}^{R_{B}}(t)$ indicates the probability of node $i$ has accepted feature $B$ at time step $t . h^{B A}$ is defined as the influence factor of feature $B$ to $A$, where $h^{B A}>0$ is called the cooperative factor when $A$ and $B$ belong to the same type of feature, while $h^{B A}<0$ is called the competitive factor when $A$ and $B$ belong to different type of feature. In this way, $r_{i}^{A}(t)$ represents the integrated impact of all the other features to $A$ at time step $t$. Specifically, $r_{i}^{A}(t)=0$ means feature $A$ diffuses from $j$ to $i$ with transmissibility $\phi_{j i}^{A}(t)=\frac{1}{1+e^{-\beta_{j i}^{A}(t)}}$ at time $t$ and do not be affected by other features. When $r_{i}^{A}(t)>0\left(r_{i}^{A}(t)<0\right)$, the influence of cooperative features regarding $A$ is larger (smaller) than the competitive features, as a consequence $i$ has larger (smaller) probability to accept the feature $A$. In addition, the sigmoid function-based representation keeps the transmissibility always in the correct range of $\left(\begin{array}{ll}0 & 1\end{array}\right)$.

We adopt the microscopic Markov chain approach (MMCA) for the evolution of the CCMF. Specifically, take the evolution of feature $A$, for example. It's MMCA equations are given by

$$
\begin{aligned}
& p_{i}^{S_{A}}(t+1)=p_{i}^{S_{A}}(t) q_{i}^{A}(t), \\
& p_{i}^{I_{A}}(t+1)=p_{i}^{S_{A}}(t)\left(1-q_{i}^{A}(t)\right)+p_{i}^{I_{A}}(t)\left(1-\varphi_{i}^{A}(t)\right), \\
& p_{i}^{R_{A}}(t+1)=p_{i}^{I_{A}}(t) \varphi_{i}^{A}(t)+p_{i}^{R_{A}}(t),
\end{aligned}
$$

where

$$
\begin{aligned}
q_{i}^{A}(t) & =\prod_{j \in \Gamma_{i}^{A}}\left(1-p_{j}^{I_{A}}(t) \phi_{j i}^{A}(t)\right) \\
& =\prod_{j \in \Gamma_{i}^{A}}\left(1-p_{j}^{I_{A}}(t) \frac{1}{1+e^{-\left(\beta_{j i}^{A}(t)+\alpha^{A} \sum_{B \in \triangle_{B}, B \neq A}\left(p_{i}^{I_{B}}(t)+p_{i}^{R_{B}}(t)\right) h^{B A}\right)}}\right) .
\end{aligned}
$$

$q_{i}^{A}(t)$ indicates the probability that node $i$ is not infected by any $I_{A}$ neighbors at time step $t$. Note that the above similar definitions and presentations apply to other features.

After the diffusions of all features reaching their stable status (e.g. at time $T$ ), the outcomes of the event $\mathbb{E}$ can be calculated through a decision-making function. We say node $i$ is $\mathbb{E}$-active if

$$
\sum_{A \in \Delta_{B}} w_{i}^{A} p_{i}^{R_{A}}(T) \geq \theta_{i}
$$

Otherwise, $i$ is $\mathbb{E}$-deactivated. $\theta_{i}$ is the decision threshold of node $i$. $w_{i}^{A}$ indicates the weight of feature $A$ for $i$ and $w_{i}^{A}>0$ when $A$ is positive feature, otherwise $w_{i}^{A}<0$. Then the total number of $\mathbb{E}$-active nodes is given by

$$
O=\sum_{i \in V}\left|\sum_{A \in \Delta_{\mathbb{B}}} w_{i}^{A} p_{i}^{R_{A}}(T) \geq \theta_{i}\right| .
$$

where $|X|=1$ if $X$ holds, otherwise $|X|=0$.

The above framework and analytic theory of CCMF is universal and have many advantages. It is suitable for the coevolution of any number of features and computationally efficient without state space explosion issue. The sigmoid function-based expression of transmissibility cleverly and reasonably integrates the impacts of the cooperative and competitive relationships between features, and also keeps its basic attribute at the same time. In addition, such expression allows the diffusions of different features following different subdiffusion dynamics.

\section{Optimal DePloyment of CONTROL Resource}

The identification of "supernodes" under the constrain of limited resource is of prime significance in diverse contexts, ranging from the suppression of undesired diffusion and the promotion of beneficial diffusion. To maximize the outcomes of the event in CCMF (due to the similarity of the maximization and minimization of the CCMF under the following proposed control strategy, we here only discuss the maximization problem of the CCMF), three solution can be adopted: purely identify the "superblocks" to minimize the diffusion of negative features, purely identify the "superspreaders" to maximize the diffusion of positive features, and maximize the diffusion of positive features and minimize the diffusion of negative features simultaneously. In this paper, we focus on the third solution due to its innovation and difficulty and the potential best performance.

Therefore, the control objective regarding CCMF is to maximize

$$
O=\sum_{i \in V}\left|\sum_{A \in \Delta_{\mathbb{E}}} w_{i}^{A} p_{i}^{R_{A}}(T) \geq \theta_{i}\right| .
$$

However, in order to make the objective function Eq. (9) be more appropriately adapted to our optimization algorithm, a 
approximate representation of Eq. (9) is given by

$$
\tilde{O}=\sum_{i \in V} \frac{1}{1+e^{-\tau\left(\sum_{A \in \triangle_{\mathcal{E}}} w_{i}^{A} p_{i}^{R_{A}}(T)-\theta_{i}\right)}} .
$$

$\tau$ is a tunable parameter and $\tau>0$. Obviously, we have $\lim _{\tau \rightarrow \infty} \tilde{O}=O$.

To maximize the CCMF by maximizing the diffusion of positive features and minimizing the diffusion of negative features simultaneously, two control mechanisms respectively corresponding to the identification of "superspreaders" of the positive feature and "superblocks" of the negative feature can be adopted which are given by

$$
i^{S_{A}}(t) \stackrel{v_{i}^{A}(t)}{\longrightarrow} i^{I_{A}}(t+1) \text { if } A \text { is positive feature }
$$

and

$$
i^{S_{B}}(t) \stackrel{m_{i}^{B}(t)}{\longrightarrow} i^{M_{B}}(t+1) \text { if } B \text { is negative feature. }
$$

Here, a novel state Immunized (M) is introduced which indicates the node is immunized against the feature and will not be infected by the feature. $v_{i}^{A}(t)$ and $m_{i}^{B}(t)$ refer to the transition probabilities of node $i$ changed from $S_{A}$ state to $I_{A}$ state and $S_{B}$ to $M_{B}$ state at time $t$ via external control respectively. Therefore, identifying the "superspreaders" and "superblocks" is converted to construct the optimal deployment of $\left\{v_{i}^{A}(t), m_{i}^{B}(t)\right\}_{i, t, A, B}$.

In this sense, the MMCA equations of the CCMF combined with the control are given by

- If $A$ is a positive feature:

$$
\begin{gathered}
p_{i}^{S_{A}}(t+1)=p_{i}^{S_{A}}(t)\left(1-v_{i}^{A}(t)\right) q_{i}^{A}(t), \\
p_{i}^{I_{A}}(t+1)=p_{i}^{S_{A}}(t)\left(1-\left(1-v_{i}^{A}(t)\right) q_{i}^{A}(t)\right)+p_{i}^{I_{A}}(t)\left(1-\varphi_{i}^{A}(t)\right), \\
p_{i}^{R_{A}}(t+1)=p_{i}^{I_{A}}(t) \varphi_{i}^{A}(t)+p_{i}^{R_{A}}(t),
\end{gathered}
$$

- If $B$ is a negative feature:

$$
\begin{aligned}
& p_{i}^{S_{B}}(t+1)=p_{i}^{S_{B}}(t)\left(1-m_{i}^{B}(t)\right) q_{i}^{B}(t), \\
& p_{i}^{I_{B}}(t+1)=p_{i}^{S_{B}}(t)\left(1-m_{i}^{B}(t)\right)\left(1-q_{i}^{B}(t)\right)+p_{i}^{I_{B}}(t)\left(1-\varphi_{i}^{B}(t)\right), \\
& p_{i}^{R_{B}}(t+1)=p_{i}^{R_{B}}(t)+p_{i}^{I_{B}}(t) \varphi_{i}^{B}(t), \\
& p_{i}^{M_{B}}(t+1)=p_{i}^{M_{B}}(t)+p_{i}^{S_{B}}(t) m_{i}^{B}(t),
\end{aligned}
$$

In addition, the control sources are not fully available and may change with time, therefore we define the available total control resource for $v_{i}^{A}(t)$ and $m_{i}^{B}(t)$ at time $t$ as

$$
\sum_{i} v_{i}^{A}(t) \leq \mathcal{B}_{v}^{A}(t)
$$

and

$$
\sum_{i} m_{i}^{B}(t) \leq \mathcal{B}_{m}^{B}(t)
$$

The $v_{i}^{A}(t)$ and $m_{i}^{B}(t)$ may also have their own variation ranges due to the external or internal constraints. That is, $v_{i}^{A}(t)$ and $m_{i}^{B}(t)$ can increase up to a certain maximum or decrease down to a certain minimum, i.e.,

$$
\underline{v_{i}^{A}(t)} \leq v_{i}^{A}(t) \leq \overline{v_{i}^{A}(t)}
$$

and

$$
\underline{m_{i}^{B}(t)} \leq m_{i}^{B}(t) \leq \overline{m_{i}^{B}(t)} .
$$

We now could present the formal definition of optimal deployment of control resource for CCMF

Problem Definition: Given evolutionary process of CCMF (e.g. Eq. (11-17)) and the constrains of control resource (e.g. Eq. (18-21)), the optimal control of CCMF is to construct the optimal deployment of $\left\{v_{i}^{A}(t), m_{i}^{B}(t)\right\}_{i, t, A, B}$ to maximize $\tilde{O}$.

From the Problem Definition, it can be found that our proposed optimal control problem of CCMF is universal, targeting arbitrary time window rather than just the initial time, considering both the network topology and diffusion dynamics, containing the optimization of maximization and minimization problems simultaneously.

In order to solve the optimal control problem of CCMF, we propose an optimization method, called OCCMF (Optimization of CCMF), based on the forward-backward propagation theory [26]. OCCMF is initialized by constructing Lagrangian formulation of our optimal control problem of the CCMF which is given by Eq. (22), where " $\lambda_{y}^{X}(t)$ " are the Lagrange multipliers. The first term of r.h.s. of Eq. (22) is the objective function. The second and third terms respectively correspond to the positive features and the negative features, each of which contains the resource constraints and the dynamical model constraints. The $\varepsilon$ is a small regularization parameter chosen to minimize the impact on the objective functions in the regime of allowed values of $v_{i}^{A}(t)$ and $m_{i}^{A}(t)$, away from their borders. Setting the derivatives of $\mathcal{L}$ with respect to $\left\{v_{i}^{A}(t)\right.$, $\left.p_{i}^{S_{A}}(t), p_{i}^{I_{A}}(t), p_{i}^{R_{A}}(t), q_{i}^{A}(t), r_{i}^{A}(t)\right\}_{i, t, A \in \Delta_{\mathrm{E}}^{+}}$and $\left\{m_{i}^{B}(t), p_{i}^{S_{B}}(t)\right.$, $\left.p_{i}^{I_{B}}(t), p_{i}^{R_{B}}(t), p_{i}^{M_{B}}(t), q_{i}^{B}(t), r_{i}^{B}(t)\right\}_{i, t, B \in \Delta_{\mathrm{B}}^{-}}$to zero, we obtain Eqs. (23) and (24).

Then we perform the forward-backward propagation iteration to derive the optimal deployment of $\left\{v_{i}^{A}(t), m_{i}^{B}(t)\right\}_{i, t, A, B}$. The processes are given by

(1) $\left\{v_{i}^{A}(t), m_{i}^{B}(t)\right\}_{i, t, A, B}$ are initialized to be arbitrary values. Let Count $=0$.

(2) Start from the given initial values of $\left\{p_{i}^{S_{A}}(0), p_{i}^{I_{A}}(0)\right.$, $\left.p_{i}^{R_{A}}(0)\right\}_{i, A \in \Delta_{\mathrm{B}}^{+}}$and $\left\{p_{i}^{S_{B}}(0), p_{i}^{I_{B}}(0), p_{i}^{R_{B}}(0), p_{i}^{M_{B}}(0)\right\}_{i, B \in \Delta_{\mathrm{B}}^{-}}$, propagate the MMCA Eqs. 11-17 forward, up to the horizon $T$ (the diffusions of all features have reached their stable status at time $T)$. We obtain the values of $\left\{p_{i}^{S_{A}}(t), p_{i}^{I_{A}}(t)\right.$, $\left.p_{i}^{R_{A}}(t), q_{i}^{A}(t) r_{i}^{A}(t)\right\}_{i, t, A \in \Delta_{\mathbb{E}}^{+}}$and $\left\{p_{i}^{S_{B}}(t), p_{i}^{I_{B}}(t), p_{i}^{R_{B}}(t), p_{i}^{M_{B}}(t)\right.$, $\left.q_{i}^{B}(t) r_{i}^{B}(t)\right\}_{i, t, B \in \Delta_{\mathbb{E}}^{-}}$.

(3) In turn calculate the boundary values of Lagrange multipliers at time $T$ 


$$
\begin{aligned}
& \mathcal{L}=\sum_{i} \frac{1}{1+e^{-\tau\left(\sum_{C \in \Delta} w_{i}^{C} p_{i}^{R_{C}}(T)-\theta_{i}\right)}} \\
& +\sum_{A \in \Delta_{\mathrm{B}}^{+}}\left[\sum_{t=0}^{T-1} \lambda_{v}^{A}(t)\left(\sum_{i \in V} v_{i}^{A}(t)-\mathcal{B}_{v}^{A}(t)\right)+\varepsilon \sum_{t=0}^{T-1} \sum_{i \in V}\left(\log \left(v_{i}^{A}(t)-\underline{v_{i}^{A}(t)}\right)+\log \left(\overline{v_{i}^{A}(t)}-v_{i}^{A}(t)\right)\right)\right. \\
& +\sum_{t=0}^{T-1} \sum_{i \in V} \lambda_{i}^{S_{A}}(t+1)\left(p_{i}^{S_{A}}(t+1)-p_{i}^{S_{A}}(t)\left(1-v_{i}^{A}(t)\right) q_{i}^{A}(t)\right) \\
& +\sum_{t=0}^{T-1} \sum_{i \in V} \lambda_{i}^{I_{A}}(t+1)\left(p_{i}^{I_{A}}(t+1)-p_{i}^{S_{A}}(t)\left(1-\left(1-v_{i}^{A}(t)\right) q_{i}^{A}(t)\right)-p_{i}^{I_{A}}(t)\left(1-\varphi_{i}^{A}(t)\right)\right) \\
& +\sum_{t=0}^{T-1} \sum_{i \in V} \lambda_{i}^{R_{A}}(t+1)\left(p_{i}^{R_{A}}(t+1)-p_{i}^{I_{A}}(t) \varphi_{i}^{A}(t)-p_{i}^{R_{A}}(t)\right) \\
& +\sum_{t=0}^{T} \sum_{i \in V} \lambda_{i}^{q^{A}}(t)\left(q_{i}^{A}(t)-\prod_{j \in \Gamma_{i}^{A}}\left(1-p_{j}^{I_{A}}(t) \frac{1}{1+e^{-\left(\beta_{j i}^{A}(t)+r_{i}^{A}(t)\right)}}\right)\right) \\
& \left.+\sum_{t=0}^{T} \sum_{i \in V} \lambda_{i}^{r^{A}}(t)\left(r_{i}^{A}(t)-\sum_{C \neq A}\left(p_{i}^{I_{C}}(t)+p_{i}^{R_{C}}(t)\right) h^{C A}\right)\right] \\
& +\sum_{B \in \Delta_{\mathrm{B}}^{-}}\left[\sum_{t=0}^{T-1} \lambda_{m}^{B}(t)\left(\sum_{i \in V} m_{i}^{B}(t)-\mathcal{B}_{m}^{B}(t)\right)+\varepsilon \sum_{t=0}^{T-1} \sum_{i \in V}\left(\log \left(m_{i}^{B}(t)-\underline{m_{i}^{B}(t)}\right)+\log \left(\overline{m_{i}^{B}(t)}-m_{i}^{B}(t)\right)\right)\right. \\
& +\sum_{t=0}^{T-1} \sum_{i \in V} \lambda_{i}^{S_{B}}(t+1)\left(p_{i}^{S_{B}}(t+1)-p_{i}^{S_{B}}(t)\left(1-m_{i}^{B}(t)\right) q_{i}^{B}(t)\right) \\
& +\sum_{t=0}^{T-1} \sum_{i \in V} \lambda_{i}^{I_{B}}(t+1)\left(p_{i}^{I_{B}}(t+1)-p_{i}^{S_{B}}(t)\left(1-m_{i}^{B}(t)\right)\left(1-q_{i}^{B}(t)\right)-p_{i}^{I_{B}}(t)\left(1-\varphi_{i}^{B}(t)\right)\right) \\
& +\sum_{t=0}^{T-1} \sum_{i \in V} \lambda_{i}^{R_{B}}(t+1)\left(p_{i}^{R_{B}}(t+1)-p_{i}^{I_{B}}(t) \varphi_{i}^{B}(t)-p_{i}^{R_{B}}(t)\right) \\
& +\sum_{t=0}^{T-1} \sum_{i \in V} \lambda_{i}^{M_{B}}(t+1)\left(p_{i}^{M_{B}}(t+1)-p_{i}^{M_{B}}(t)-p_{i}^{S_{B}}(t) m_{i}^{B}(t)\right) \\
& +\sum_{t=0}^{T} \sum_{i \in V} \lambda_{i}^{q^{B}}(t)\left(q_{i}^{B}(t)-\prod_{j \in \Gamma_{i}^{B}}\left(1-p_{j}^{I_{B}}(t) \frac{1}{1+e^{-\left(\beta_{j i}^{B}(t)+r_{i}^{B}(t)\right)}}\right)\right) \\
& \left.+\sum_{t=0}^{T} \sum_{i \in V} \lambda_{i}^{r^{B}}(t)\left(r_{i}^{B}(t)-\sum_{C \neq B}\left(p_{i}^{I_{C}}(t)+p_{i}^{R_{C}}(t)\right) h^{C B}\right)\right]
\end{aligned}
$$

- Eq. (23b) and Eq. (24b) assign $\lambda_{i}^{S_{A}}(T)=0$ and $\lambda_{i}^{S_{B}}(T)=0$ respectively.

- Eq. (24e) gives $\lambda_{i}^{M_{B}}(T)=0$;

- Eq. (23e) and Eq. (24f) give $\lambda_{i}^{q^{A}}(T)=0$ and $\lambda_{i}^{q^{B}}(T)=0$ respectively;

- Eq. (23f) and Eq. (24g) assign $\lambda_{i}^{r^{A}}(T)=0$ and $\lambda_{i}^{r^{B}}(T)=0$ based on $\lambda_{i}^{q^{A}}(T)$ and $\lambda_{i}^{q^{B}}(T)$ respectively;

- Eq. (23d) assigns $\lambda_{i}^{R_{A}}(T)=-\frac{\tau w_{i}^{A} e^{-\tau\left(\Sigma_{A \in \triangle_{\mathbb{B}}} w_{i}^{A} p_{i}^{R_{A}}(T)-\theta_{i}\right)}}{\left(1+e^{-\tau\left(\Sigma_{A \in \triangle_{\mathbb{E}}} w_{i}^{A} p_{i}^{R_{A}}(T)-\theta_{i}\right)}\right)^{2}}$ and Eq. (24d) assigns $\lambda_{i}^{R_{B}}(T)=-\frac{\tau w_{i}^{B} e^{-\tau \tau\left(\Sigma_{A \in A_{B}} w_{i}^{A} p_{i}^{R_{A}}(T)-\theta_{i}\right)}}{\left(1+e^{-\tau\left(\Sigma_{A \in \mathbb{A}_{\mathbb{B}}} w_{i}^{A} p_{i}^{R_{A}}(T)-\theta_{i}\right)}\right)^{2}}$ respectively;

- Eq. (23c) and Eq. (24c) assign $\lambda_{i}^{I_{A}}(T)=0$ and $\lambda_{i}^{I_{B}}(T)=0$ respectively;

- From Eq. (23a), we have

$$
\begin{aligned}
& v_{i}^{A}(T-1)= \\
& \frac{\lambda_{v}^{A}(T-1)+L v_{i}^{A}(T-1)-2 \varepsilon \pm \sqrt{\left(\lambda_{v}^{A}(T-1)+L v_{i}^{A}(T-1)\right)^{2}+4 \varepsilon^{2}}}{2\left(\lambda_{v}^{A}(T-1)+L v_{i}^{A}(T-1)\right)},
\end{aligned}
$$

where

$$
\begin{aligned}
& L v_{i}^{A}(T-1)= \\
& \lambda_{i}^{S_{A}}(T) p_{i}^{S_{A}}(T-1) q_{i}^{A}(T-1)-\lambda_{i}^{I_{A}}(T) p_{i}^{S_{A}}(T-1) q_{i}^{A}(T-1) .
\end{aligned}
$$

Based on the resource constraint $\sum_{i} v_{i}^{A}(T-1)=\mathcal{B}_{v}^{A}(T-1)$, we could numerically obtain $\lambda_{v}^{A}(T-1)$ and hence obtain 


$$
\begin{aligned}
& \partial \mathcal{L} / \partial v_{i}^{A}(t)=\lambda_{v}^{A}(t)+\frac{\varepsilon}{v_{i}^{A}(t)-\underline{v_{i}^{A}(t)}}-\frac{\varepsilon}{\overline{v_{i}^{A}(t)}-v_{i}^{A}(t)}+\left(\lambda_{i}^{S_{A}}(t+1) p_{i}^{S_{A}}(t) q_{i}^{A}(t)-\lambda_{i}^{I_{A}}(t+1) p_{i}^{S_{A}}(t) q_{i}^{A}(t)\right)|t \neq T|=0 \\
& \partial \mathcal{L} / \partial p_{i}^{S_{A}}(t)=\lambda_{i}^{S_{A}}(t)+\left(-\lambda_{i}^{S_{A}}(t+1)\left(1-v_{i}^{A}(t)\right) q_{i}^{A}(t)-\lambda_{i}^{I_{A}}(t+1)\left(1-\left(1-v_{i}^{A}(t)\right) q_{i}^{A}(t)\right)\right)|t \neq T|=0 \\
& \partial \mathcal{L} / \partial p_{i}^{I_{A}}(t)=\lambda_{i}^{I_{A}}(t)+\left(-\lambda_{i}^{I_{A}}(t+1)\left(1-\varphi_{i}^{A}(t)\right)-\lambda_{i}^{R_{A}}(t+1) \varphi_{i}^{A}(t)\right)|t \neq T| \\
& +\sum_{j \in \Gamma_{i}^{A}} \lambda_{j}^{q^{A}}(t) \frac{1}{1+e^{-\left(\beta_{i j}^{A}(t)+r_{j}^{A}(t)\right)}} \prod_{k \in \Gamma_{j}^{A}, k \neq i}\left(1-p_{k}^{I_{A}}(t) \frac{1}{1+e^{-\left(\beta_{k j}^{A}(t)+r_{j}^{A}(t)\right)}}\right)-\sum_{C \neq A} \lambda_{i}^{r^{C}}(t) h^{A C}=0 \\
& \partial \mathcal{L} / \partial p_{i}^{R_{A}}(t)=\frac{\tau w_{i}^{A} e^{-\tau\left(\sum_{A} w_{i}^{A} p_{i}^{R_{A}}(T)-\theta_{i}\right)}}{\left(1+e^{-\tau\left(\sum_{A} w_{i}^{A} p_{i}^{R_{A}}(T)-\theta_{i}\right)}\right)^{2}}[t=T]+\lambda_{i}^{R_{A}}(t)-\lambda_{i}^{R_{A}}(t+1)|t \neq T|-\sum_{C \neq A} \lambda_{i}^{r^{C}}(t) h^{A C}=0 \\
& \left.\partial \mathcal{L} / \partial q_{i}^{A}(t)=\left(-\lambda_{i}^{S_{A}}(t+1) p_{i}^{S_{A}}(t)\right)\left(1-v_{i}^{A}(t)\right)+\lambda_{i}^{I_{A}}(t+1) p_{i}^{S_{A}}(t)\left(1-v_{i}^{A}(t)\right)\right)|t \neq T|+\lambda_{i}^{q^{A}}(t)=0 \\
& \partial \mathcal{L} / \partial r_{i}^{A}(t)=\lambda_{i}^{r^{A}}(t)+\sum_{j \in \Gamma_{i}^{A}} p_{j}^{I_{A}}(t) e^{-\left(\beta_{j i}^{A}(t)+r_{i}^{A}(t)\right)} \frac{1}{\left(1+e^{-\left(\beta_{j i}^{A}(t)+r_{i}^{A}(t)\right)}\right)^{2}} \lambda_{i}^{q^{A}}(t) \prod_{k \in \Gamma_{i}^{A}, k \neq j}\left(1-p_{k}^{I_{A}}(t) \frac{1}{1+e^{-\left(\beta_{k i}^{A}(t)+r_{i}^{A}(t)\right)}}\right)=0
\end{aligned}
$$

$$
\begin{aligned}
\partial \mathcal{L} / \partial m_{i}^{B}(t)= & \lambda_{m}^{B}(t)+\frac{\varepsilon}{m_{i}^{B}(t)-\frac{m_{i}^{B}(t)}{S_{i}^{B}(t)}-m_{i}^{B}(t)} \\
& +\left(\lambda_{i}^{S_{B}}(t+1) p_{i}^{S_{B}}(t) q_{i}^{B}(t)+\lambda_{i}^{I_{B}}(t+1) p_{i}^{S_{B}}(t)\left(1-q_{i}^{B}(t)\right)-\lambda_{i}^{M_{B}}(t+1) p_{i}^{S_{B}}(t)\right)|t \neq T|=0 \\
\partial \mathcal{L} / \partial p_{i}^{S_{B}}(t)= & \lambda_{i}^{S_{B}}(t)+\left(-\lambda_{i}^{S_{B}}(t+1)\left(1-m_{i}^{B}(t)\right) q_{i}^{B}(t)-\lambda_{i}^{I_{B}}(t+1)\left(1-m_{i}^{B}(t)\right)\left(1-q_{i}^{B}(t)\right)-\lambda_{i}^{M_{B}}(t+1) m_{i}^{B}(t)\right)|t \neq T|=0 \\
\partial \mathcal{L} / \partial p_{i}^{I_{B}}(t)= & \lambda_{i}^{I_{B}}(t)+\left(-\lambda_{i}^{I_{B}}(t+1)\left(1-\varphi_{i}^{B}(t)\right)-\lambda_{i}^{R_{B}}(t+1) \varphi_{i}^{B}(t)\right)|t \neq T| \\
& +\sum_{j \in \Gamma_{i}^{B}} \lambda_{j}^{q^{B}}(t) \frac{1}{1+e^{-\left(\beta_{i j}^{B}(t)+r_{j}^{B}(t)\right)}} \prod_{k \in \Gamma_{j}^{B}, k \neq i}\left(1-p_{k}^{I_{B}}(t) \frac{1}{\left.1+e^{-\left(\beta_{k j}^{B}(t)+r_{j}^{B}(t)\right)}\right)-\sum_{C \neq B} \lambda_{i}^{r^{C}}(t) h^{B C}=0}\right. \\
\partial \mathcal{L} / \partial p_{i}^{R_{B}}(t)= & \frac{\tau w_{i}^{B} e^{-\tau\left(\sum_{B} w_{i}^{B} p_{i}^{R_{B}}(T)-\theta_{i}\right)}}{\left(1+e^{-\tau\left(\sum_{B} w_{i}^{B} p_{i}^{R_{B}}(T)-\theta_{i}\right)}\right)^{2}}[t=T]+\lambda_{i}^{R_{B}}(t)-\lambda_{i}^{R_{B}}(t+1)|t \neq T|-\sum_{C \neq B} \lambda_{i}^{r^{C}}(t) h^{B C}=0 \\
\partial \mathcal{L} / \partial p_{i}^{M_{B}}(t)= & \lambda_{i}^{M_{B}}(t)-\lambda_{i}^{M_{B}}(t+1)|t \neq T|=0 \\
\partial \mathcal{L} / \partial q_{i}^{B}(t)= & \left(-\lambda_{i}^{S_{B}}(t+1) p_{i}^{S_{B}}(t)\left(1-m_{i}^{B}(t)\right)+\lambda_{i}^{I_{B}}(t+1) p_{i}^{S_{B}}(t)\left(1-m_{i}^{B}(t)\right)\right)|t \neq T|+\lambda_{i}^{q^{B}}(t)=0 \\
\partial \mathcal{L} / \partial r_{i}^{B}(t)= & \lambda_{i}^{r^{B}}(t)+\sum_{j \in \Gamma_{i}^{B}} p_{j}^{I_{B}}(t) e^{-\left(\beta_{j i}^{B}(t)+r_{i}^{B}(t)\right)} \frac{1}{\left(1+e^{-\left(\beta_{j i}^{B}(t)+r_{i}^{B}(t)\right)}\right)^{2}} \lambda_{i}^{q^{B}}(t) \prod_{k \in \Gamma_{i}^{B}, k \neq j}\left(1-p_{k}^{I_{B}}(t) \frac{1}{\left.1+e^{-\left(\beta_{k i}^{B}(t)+r_{i}^{B}(t)\right)}\right)=0}\right.
\end{aligned}
$$

$v_{i}^{A}(T-1)$. Then in the same way, we could obtain $\lambda_{m}^{B}(T-1)$ and $m_{i}^{B}(T-1)$ based on Eq. (24a).

(3) Use the above boundary values of Lagrange multipliers and $v_{i}^{A}(T-1)_{i, A \in \Delta_{\mathrm{B}}^{+}}$and $m_{i}^{A}(T-1)_{i, B \in \Delta_{\mathrm{B}}^{-}}$, propagate Eqs. (29a)(29h) backward to calculate the Lagrange multipliers and $m_{i}^{A}(t)$ and $v_{i}^{A}(t)$ regarding all nodes, times and features step by step.

(4) Count=Count +1 . If Count equals to the fixed number (the fixed number is 8 in this paper), the algorithm terminates, otherwise go back to Step (2).

The OCCMF is designed based on the MMCA equations of the CCMF and thus is also computationally efficient. It derive the optimal deployment of $\left\{v_{i}^{A}(t), m_{i}^{B}(t)\right\}_{i, t, A, B}$ by considering both the network topology, the diffusion dynamics and the interplays between features, and thus the result is comprehensively.

\section{Results}

Simulation experiments are performed in this section. We first present a CCMF model with three features $\{A, B, C\}$ regarding event $\mathbb{E}$, where $A, B \in \Delta_{\mathbb{E}}^{+}$and $C \in \Delta_{\mathbb{E}}^{-}$. The three features diffuse following the SIR model. The underlying network is a multiplex network with three layers $G=\left\{G^{A}, G^{B}, G^{C}\right\}$. The framework of network and features is shown in Fig. 1. Unless otherwise specified, the other corresponding parameters of diffusion model and the network topology are consistent with that in above two sections. 


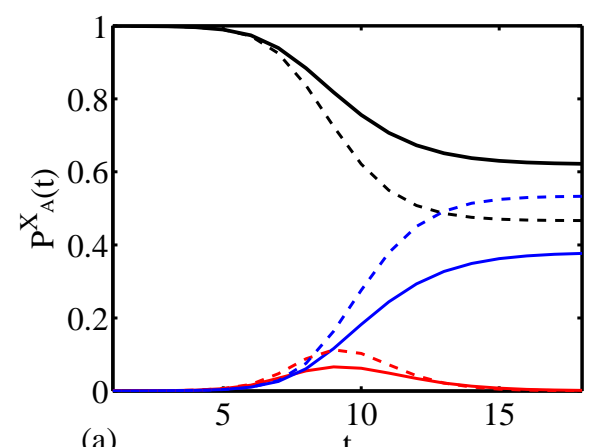

(a)

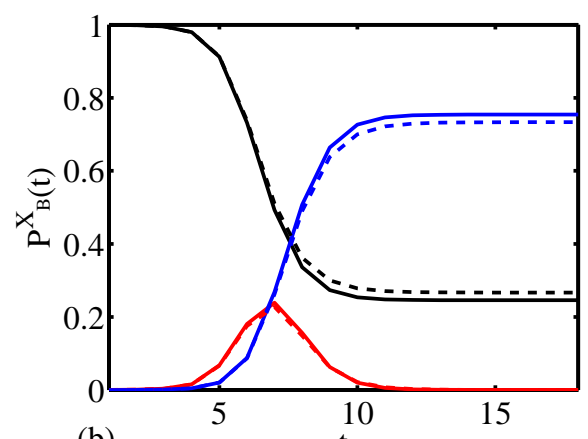

(b)

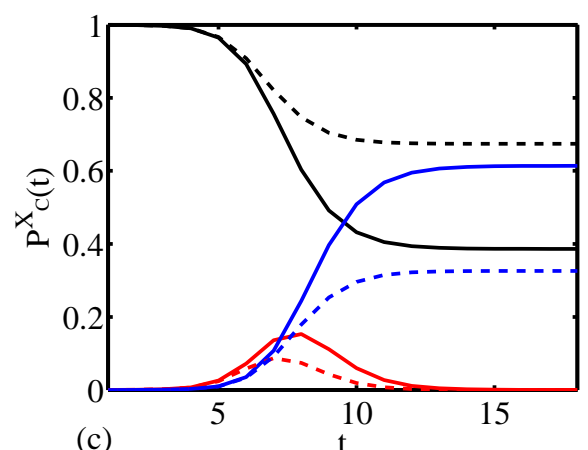

(c)

Fig. 2. The densities of susceptible nodes (black line), infected nodes (red line) and recovered nodes (blue line) versus the time. Panels (a), (b) and (c) correspond to features $A, B$ and $C$ respectively.

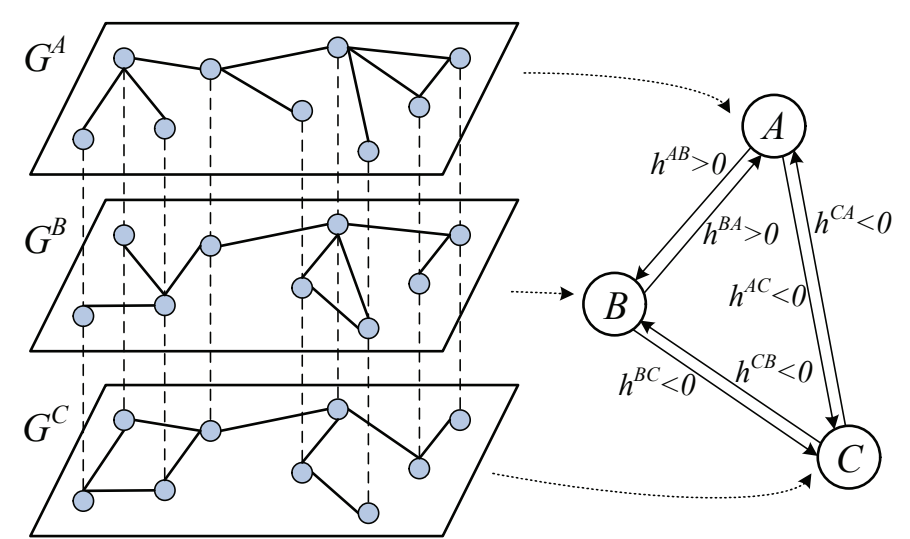

Fig. 1. The framework of network and features in CCMF.

Fig. 2 shows the difference of the MFD with (the dashed lines) and without (the solid lines) the interactions between features. We assume the diffusions of the features have same dynamical parameters, such as $\beta_{j i}^{Y}(t)=-1$ and $\varphi_{i}^{Y}(t)=1$ for all nodes $i$ and $j$, feature $Y$ and time $t$. Each layers of the underlying multiplex network are all scale-free networks. The only difference is the average degrees of the layers which are $\left\langle k_{A}\right\rangle=4,\left\langle k_{B}\right\rangle=8$ and $\left\langle k_{C}\right\rangle=6 . P^{X_{Y}}(t)=\sum_{i} p_{i}^{X_{Y}}(t) / N$ indicates the density of $X_{Y}$ state nodes at time $t$. It can be found that the influence of cooperation and competition between features in MFD is pretty obvious. For example, feature $A$ outbreaks easier and diffuses larger scale than it diffuses independently, due to the strong cooperation from $B$ (large average degree) and weak competition from $C$ (small average degree). On the contrary, the outbreak size of $B$ becomes smaller than it diffuses independently, since the influence of cooperation of $A$ is weaker than the competition of $C$. The feature $C$ is significantly suppressed since it just gets competitions from other features.

In essence, the effect of the cooperation and competition between features is mainly featured in changing the transmissibility of feature. We show such effect through two random selected nodes in Fig. 3. One sees that the transmissibility of feature $Y$ between node $i$ and its neighbors $\left(\phi_{j i}^{Y}(t)=\phi_{i}^{Y}(t)\right.$ for $\forall j$ ) changes with $r_{i}^{Y}(t)$ in same trend. The transmissibility gets bigger when the $r_{i}^{Y}>0$. That is the nodes will be more easier to accepted feature $Y$ when it has accepted more same type of other features, and vice versa.
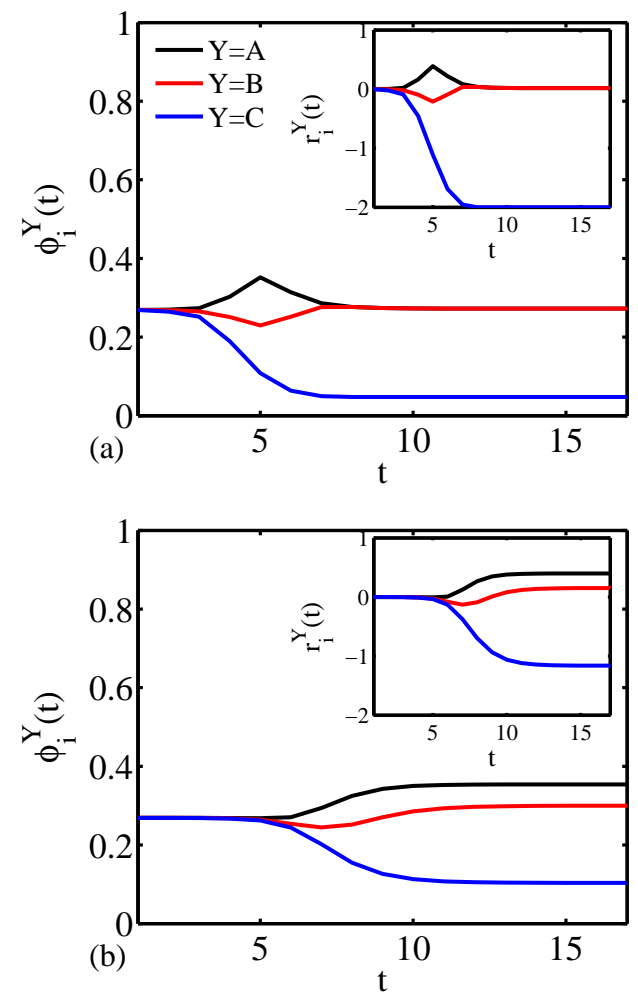

Fig. 3. The evolutions of $\phi_{i}^{Y}(t)$ and $r_{i}^{Y}(t)$ of two nodes, $i=2$ in panel (a) and $i=2000$ in panel (b).

Fig. 4 shows the impacts of the influence factors and diffusion dynamical parameters on the outcomes of the CCMF. The color of the panel represents the final density of the $\mathbb{E}$ active nodes, i.e. $\rho=O / N$. We let the cooperative factor $h^{A B}=h^{B A}=h^{+}$and the competitive factor $h^{A C}=h^{C A}=h^{B C}=$ $h^{C B}=h^{-}$. In Fig. (4a), same parameters and network topology are used with that in Fig. 1 except $\beta_{j i}^{C}(t)$. Here, we let $\beta_{j i}^{C}(t)=$ $\beta^{C}=-0.6$ for any $i, j$ and $t$. A normal result is obtained that $\rho$ increases with the enhance of cooperation between features and decreases with the enhance of competition. However, if we change $\beta^{C}$ to -1 which indicates a smaller transmissibility of feature $C$ is adopted, an opposite result appears. As shown 

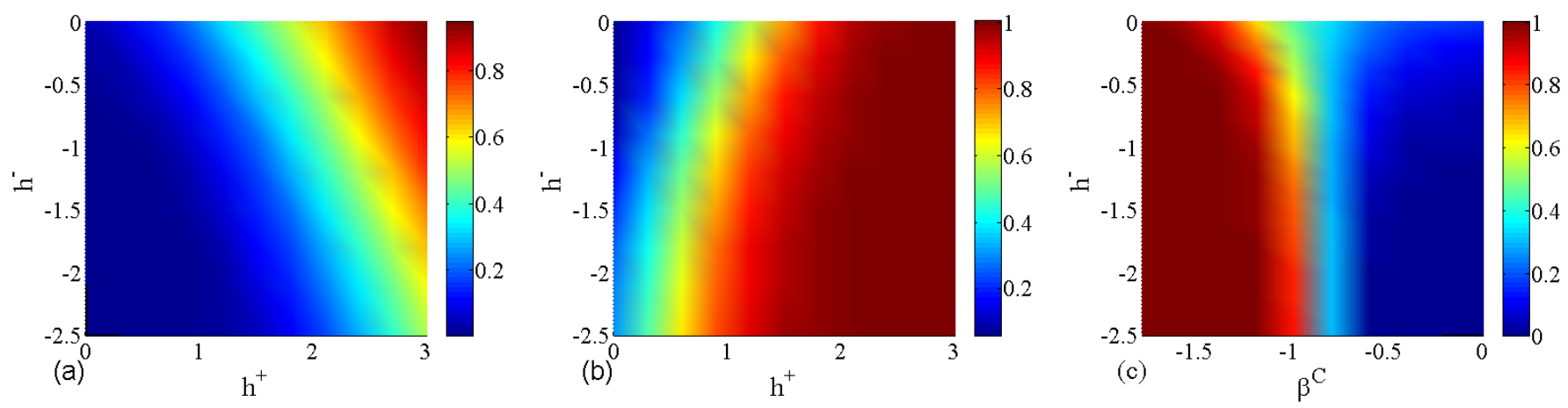

Fig. 4. Panel (a) and (b): $\rho$ versus $h^{+}$and $h^{-}$for different values of $\beta^{C}$. Panel (c): $\rho$ versus $\beta^{C}$ and $h^{-}$.

in Fig. (4b), the density of $\mathbb{E}$-active nodes $\rho$ increases with the enhance of competition, which is contrary to our intuitive cognition. In order to explain such phenomenon, we discuss the relationships of $\beta^{C}, h^{-}$and $\rho$. From Fig. (4c), an very interesting result is obtained: there is an obvious threshold of $\beta^{C}$, denoted as $\beta_{c}^{C}$, below which, $\rho$ increases with the enhance of competition, otherwise, $\rho$ decreases with the enhance of competition. If we call the $\beta^{C}$ the competition ability, an interesting conclusion can be derived: When the feature has weak competition ability, performing competition with high intensity could more easily lead to failure in competition. The high competition intensity require strong competition ability to get positive benefits.

In Fig. 5, we verify the efficiency of our proposed OCCMF optimization algorithm. OCCMF could identify the optimal deployment of $\left\{v_{i}^{A}(t), m_{i}^{B}(t)\right\}_{i, t, A, B}$ under the limited control resource simultaneously. It considers both the network topology, the diffusion dynamics and the interplays between features. Panel (a) shows the results of different control optimization for the CCMF model on both multiplex scale free networks and multiplex random networks. Here we assume that the control resources are available and unchanged at time 0 to 6 which are given by $\mathcal{B}_{v}^{A}(t)=0.005 \mathrm{~N}$ and $\mathcal{B}_{m}^{A}(t)=0.005 \mathrm{~N}$ for any $A$ and $t \in\left[\begin{array}{ll}0 & 6\end{array}\right]$. Different case is also considered in panel (b) in which the control resources change with time as shown in the inset. The dark blue bar correspond to no available control resource. The light blue bar refers to the random deployment of all control resource. The green bar only optimizes the features $A$ and $B$ via the OCCMF, feature $C$ adopts the random deployment method. Correspondingly, the orange bar only optimizes the feature $C$ via the OCCMF, features $A$ and $B$ adopts the random deployment method. The brown bar optimizes $A, B$ and $C$ via the OCCMF simultaneously. For unchanged control source in panel (a), it can be found that our OCCMF algorithm shows remarkable effect on the optimization of control resources. The $\mathbb{E}$-active nodes obtained from OCCMF is about 5 times the final density of the random deployment method for SF network, and about 2.5 times for ER network. In addition, the simultaneous optimization of $A, B$ and $C$ is also remarkably better than the optimization of parts of features. Panel (b) presents the outcomes of the OCCMF under changed control resources. The OCCMF is still the best which further illustrates its universality and extensive suitability.
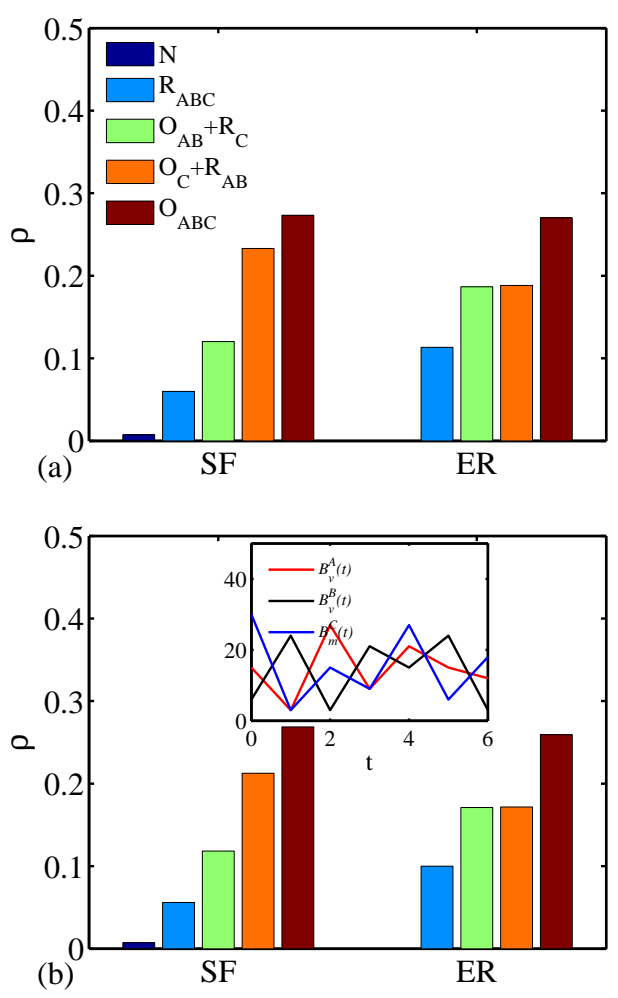

Fig. 5. The comparison of different optimization schemes.

\section{Conclusion}

The MFD well characterized the scenario where the event is determined by the diffusion of multiple features regarding the event. In this paper, we focus on MFD and introduced the cooperative relationship and the competitive relationship between features into the MFD, and proposed the model of CCMF in multiplex network. Then an MMCA was presented which is applicable and computationally efficient to modeling the evolution process of CCMF with any number of features and different sub-diffusion dynamics. By studying the impacts of influence factor and transmissibility on the CCMF, an interesting founding was obtained. That is performing high intensity competition under weak competition ability is more 
easier to result in negative effect. To optimal control the CCMF under given resource, an optimization strategy OCCMF was proposed which has universality and extensive suitability. OCCMF could optimally allocate the given control resource and could promote and suppress the network diffusion simultaneously.

\section{REFERENCES}

[1] R. Pastor-Satorras, C. Castellano, P. Van Mieghem, and A. Vespignani, "Epidemic processes in complex networks," Reviews of modern physics, vol. 87 , no. 3 , p. $925,2015$.

[2] N. Masuda, M. A. Porter, and R. Lambiotte, "Random walks and diffusion on networks," Physics reports, vol. 716, pp. 1-58, 2017.

[3] R. Gallotti, F. Valle, N. Castaldo, P. Sacco, and M. De Domenico, "Assessing the risks of infodemics in response to covid-19 epidemics," Nature Human Behaviour, vol. 4, no. 12, pp. 1285-1293, 2020.

[4] Y.-Q. Zhang, X. Li, and A. V. Vasilakos, "Spectral analysis of epidemic thresholds of temporal networks," IEEE transactions on cybernetics, vol. 50, no. 5, pp. 1965-1977, 2017.

[5] S. Peng, S. Yu, and A. Yang, "Smartphone malware and its propagation modeling: A survey," IEEE Communications Surveys $\mathcal{E}$ Tutorials, vol. 16, no. 2, pp. 925-941, 2013.

[6] S. V. Buldyrev, R. Parshani, G. Paul, H. E. Stanley, and S. Havlin, "Catastrophic cascade of failures in interdependent networks," Nature, vol. 464, no. 7291, pp. 1025-1028, 2010.

[7] C. Nowzari, V. M. Preciado, and G. J. Pappas, "Analysis and control of epidemics: A survey of spreading processes on complex networks," IEEE Control Systems Magazine, vol. 36, no. 1, pp. 26-46, 2016.

[8] Q. Zhang, L. Zhong, S. Gao, and X. Li, "Optimizing hiv interventions for multiplex social networks via partition-based random search," IEEE transactions on cybernetics, vol. 48, no. 12, pp. 3411-3419, 2018.

[9] Z. Wang, C. T. Bauch, S. Bhattacharyya, A. d'Onofrio, P. Manfredi, M. Perc, N. Perra, M. Salathé, and D. Zhao, "Statistical physics of vaccination," Physics Reports, vol. 664, pp. 1-113, 2016.

[10] S. Boccaletti, V. Latora, Y. Moreno, M. Chavez, and D.-U. Hwang, "Complex networks: Structure and dynamics," Physics reports, vol. 424 , no. 4-5, pp. 175-308, 2006.

[11] S. Xu, P. Wang, C.-X. Zhang, and J. J. Lü, "Spectral learning algorithm reveals propagation capability of complex networks," IEEE transactions on cybernetics, vol. 49, no. 12, pp. 4253-4261, 2018.

[12] R. Pastor-Satorras and A. Vespignani, "Epidemic spreading in scale-free networks," Physical review letters, vol. 86, no. 14, p. 3200, 2001

[13] G. F. de Arruda, F. A. Rodrigues, and Y. Moreno, "Fundamentals of spreading processes in single and multilayer complex networks," Physics Reports, vol. 756, pp. 1-59, 2018.

[14] G. Bianconi, Multilayer networks: structure and function. Oxford university press, 2018.

[15] X. Zhao, J. Zhou, and J.-A. Lu, "Pinning synchronization of multiplex delayed networks with stochastic perturbations," IEEE transactions on cybernetics, vol. 49, no. 12, pp. 4262-4270, 2018.

[16] D. Zhao, G. Xiao, Z. Wang, L. Wang, and L. Xu, "Minimum dominating set of multiplex networks: definition, application, and identification," IEEE Transactions on Systems, Man, and Cybernetics: Systems, DOI: 10.1109/TSMC.2020.2987163, 2020.

[17] S. Boccaletti, G. Bianconi, R. Criado, C. I. Del Genio, J. GómezGardenes, M. Romance, I. Sendina-Nadal, Z. Wang, and M. Zanin, "The structure and dynamics of multilayer networks," Physics reports, vol. 544, no. 1, pp. 1-122, 2014.

[18] M. Salehi, R. Sharma, M. Marzolla, M. Magnani, P. Siyari, and D. Montesi, "Spreading processes in multilayer networks," IEEE Transactions on Network Science and Engineering, vol. 2, no. 2, pp. 65-83, 2015.

[19] D. Zhao, L. Li, H. Peng, Q. Luo, and Y. Yang, "Multiple routes transmitted epidemics on multiplex networks," Physics Letters A, vol. 378, no. 10, pp. 770-776, 2014.

[20] C. Granell, S. Gómez, and A. Arenas, "Dynamical interplay between awareness and epidemic spreading in multiplex networks," Physical review letters, vol. 111, no. 12, p. 128701, 2013.

[21] T. Chen, J. Guo, and W. Wu, "Adaptive multi-feature budgeted profit maximization in social networks," arXiv preprint arXiv:2006.03222, 2020.

[22] J. Guo, T. Chen, and W. Wu, "A multi-feature diffusion model: Rumor blocking in social networks," IEEE/ACM Transactions on Networking, vol. 29 , no. 1, pp. 386-397, 2020.
[23] P.-Y. Chen, S.-M. Cheng, and K.-C. Chen, "Optimal control of epidemic information dissemination over networks," IEEE transactions on cybernetics, vol. 44, no. 12, pp. 2316-2328, 2014.

[24] Y. Chen, G. Paul, S. Havlin, F. Liljeros, and H. E. Stanley, "Finding a better immunization strategy," Physical review letters, vol. 101, no. 5, p. 058701, 2008.

[25] Y. Liu, H. Sanhedrai, G. Dong, L. M. Shekhtman, F. Wang, S. V. Buldyrev, and S. Havlin, "Efficient network immunization under limited knowledge," National Science Review, vol. 8, no. 1, p. nwaa229, 2021.

[26] A. Y. Lokhov and D. Saad, "Optimal deployment of resources for maximizing impact in spreading processes," Proceedings of the National Academy of Sciences, vol. 114, no. 39, pp. E8138-E8146, 2017.

[27] F. Morone and H. A. Makse, "Influence maximization in complex networks through optimal percolation," Nature, vol. 524, no. 7563, pp. 65-68, 2015.

[28] L. Lü, D. Chen, X.-L. Ren, Q.-M. Zhang, Y.-C. Zhang, and T. Zhou, "Vital nodes identification in complex networks," Physics Reports, vol. 650, pp. 1-63, 2016.

[29] S. Wang, J. Liu, and Y. Jin, "Finding influential nodes in multiplex networks using a memetic algorithm," IEEE transactions on cybernetics, vol. 51, no. 2, pp. 900-912, 2021.

[30] D. Zhao, S. Yang, X. Han, S. Zhang, and Z. Wang, "Dismantling and vertex cover of network through message passing," IEEE Transactions on Circuits and Systems II: Express Briefs, vol. 67, no. 11, pp. 27322736, 2020.

[31] H. Chen, G. Li, H. Zhang, and Z. Hou, "Optimal allocation of resources for suppressing epidemic spreading on networks," Physical Review E, vol. 96, no. 1, p. 012321, 2017.

[32] L. Ding, P. Hu, Z.-H. Guan, and T. Li, "An efficient hybrid control strategy for restraining rumor spreading," IEEE Transactions on Systems, Man, and Cybernetics: Systems, 2020.

[33] F. M. Bass, "A new product growth for model consumer durables," Management science, vol. 15, no. 5, pp. 215-227, 1969.

[34] W. Huang and C. Li, "Epidemic spreading in scale-free networks with community structure," Journal of Statistical Mechanics: Theory and Experiment, vol. 2007, no. 01, p. P01014, 2007.

[35] B. Wang, W. Chen, J. Wang, B. Zhang, Z. Zhang, and X. Qiu, "Cooperative tracking control of multiagent systems: A heterogeneous coupling network and intermittent communication framework," IEEE transactions on cybernetics, vol. 49, no. 12, pp. 4308-4320, 2018.

[36] W. Wang, Q.-H. Liu, S.-M. Cai, M. Tang, L. A. Braunstein, and H. E. Stanley, "Suppressing disease spreading by using information diffusion on multiplex networks," Scientific reports, vol. 6, no. 1, pp. 1-14, 2016.

[37] D. Zhao, L. Wang, Z. Wang, and G. Xiao, "Virus propagation and patch distribution in multiplex networks: modeling, analysis, and optimal allocation," IEEE Transactions on Information Forensics and Security, vol. 14, no. 7, pp. 1755-1767, 2018.

[38] Z. Wang, C. Xia, Z. Chen, and G. Chen, "Epidemic propagation with positive and negative preventive information in multiplex networks," IEEE transactions on cybernetics, vol. 51, no. 3, pp. 1454-1462, 2020.

[39] X. Wei, S. Chen, X. Wu, D. Ning, and J.-a. Lu, "Cooperative spreading processes in multiplex networks," Chaos: An Interdisciplinary Journal of Nonlinear Science, vol. 26, no. 6, p. 065311, 2016.

[40] X. Wei, S. Chen, X. Wu, J. Feng, and J.-a. Lu, "A unified framework of interplay between two spreading processes in multiplex networks," $E P L$ (Europhysics Letters), vol. 114, no. 2, p. 26006, 2016.

[41] Q. Li, Z. Wang, B. Wu, and Y. Xiao, "Competition and cooperation Dynamical interplay diffusion between social topic multiple messages in multiplex networks," IEEE Transactions on Computational Social Systems, vol. 6, no. 3, pp. 467-478, 2019.

[42] S. Li, D. Zhao, X. Wu, Z. Tian, A. Li, and Z. Wang, "Functional immunization of networks based on message passing," Applied Mathematics and Computation, vol. 366, p. 124728, 2020.

[43] S. Mugisha and H.-J. Zhou, "Identifying optimal targets of network attack by belief propagation," Physical Review E, vol. 94, no. 1, p. 012305, 2016.

[44] P. Clusella, P. Grassberger, F. J. Pérez-Reche, and A. Politi, "Immunization and targeted destruction of networks using explosive percolation," Physical review letters, vol. 117, no. 20, p. 208301, 2016.

[45] E. Cozzo, M. Kivelä, M. De Domenico, A. Solé, A. Arenas, S. Gómez, M. A. Porter, and Y. Moreno, "Clustering coefficients in multiplex networks," arXiv preprint arXiv:1307.6780, 2013

[46] L. Solá, M. Romance, R. Criado, J. Flores, A. García del Amo, and S. Boccaletti, "Eigenvector centrality of nodes in multiplex networks," Chaos: An Interdisciplinary Journal of Nonlinear Science, vol. 23, no. 3, p. 033131, 2013. 
[47] A. Halu, R. J. Mondragón, P. Panzarasa, and G. Bianconi, "Multiplex pagerank," PloS one, vol. 8, no. 10, p. e78293, 2013.

[48] S. Osat, A. Faqeeh, and F. Radicchi, "Optimal percolation on multiplex networks," Nature communications, vol. 8, no. 1, pp. 1-7, 2017.

[49] F. Altarelli, A. Braunstein, L. DallAsta, J. R. Wakeling, and R. Zecchina, "Containing epidemic outbreaks by message-passing techniques," Physical Review X, vol. 4, no. 2, p. 021024, 2014.

[50] M. Gong, J. Yan, B. Shen, L. Ma, and Q. Cai, "Influence maximization in social networks based on discrete particle swarm optimization," Information Sciences, vol. 367, pp. 600-614, 2016.

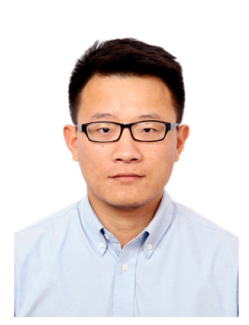

Dawei Zhao is currently a professor with the Shandong Computer Science Center (National Supercomputer Center in Jinan), China. He received the Ph.D. degree in cryptology from Beijing University of Posts and Telecommunications in 2014. His main research interests include network security, complex network and epidemic spreading dynamics.

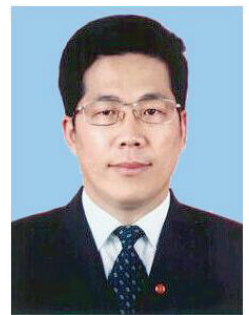

Yinglong Wang received the M.S. degree in industrial automation and the Ph.D. degree in communication and information systems from Shandong University, Jinan, China, in 1990 and 2005, respectively. $\mathrm{He}$ is currently a Research Fellow with the Shandong Computer Science Center (National Supercomputer Center in Jinan), Qilu University of Technology (Shandong Academy of Sciences), Jinan. His current research interests include wireless networks, information security, and cloud computing.

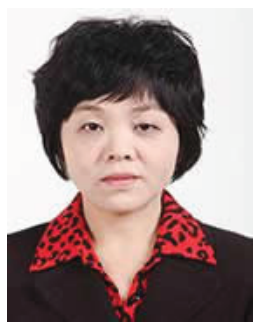

Meihong Yang received the B.S. and M.S. degrees from Shandong University, Jinan, China, in 2004 and 2007, respectively. She is currently the Technology Officer and a Professor with the Shandong Computer Science Center (National Supercomputer Center in Jinan), Jinan, China. Her research interests include cloud computing and computer networks.

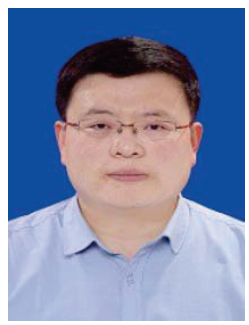

Shudong Li received M.S. degree in applied mathematics from Tongji University (China) in June 2005 and his Ph.D. degree in Information Security at Beijing University of Posts and Telecommunications (China) in July 2012. From 2013-2018, He was the postdoc of National University of Defense Technology China. Now, he is associate professor in Cyberspace Institute of Advanced Technology, Guangzhou University, China. His current research includes Big Data and its security, IoT security, information security and cryptography, the robustness of complex networks. His awards include 2015 First prize of scientific and technological progress in Hunan province, China, and 2018 First prize of Chans Chinese information processing science and technology, China. $\mathrm{He}$ won the best paper award at CIAT 2020.
Zhen Wang received the Ph.D. degree from Hong Kong Baptist University, Hong Kong, in 2014. From 2014 to 2016, he was a JSPS Senior Researcher with the Interdisciplinary Graduate School of Engineering Sciences, Kyushu University, Fukuoka, Japan. Since 2017, he has been a Full Professor with Northwestern Polytechnical University, Xi'an, China. His current research interests include network science, complex system, big data, evolutionary game theory, behavior decision and behavior recognition. Thus far, he has published more than 100 scientific papers and obtained around 8000 citations. Besides, he serves as an Editor or Academic Editor for 7 journals.

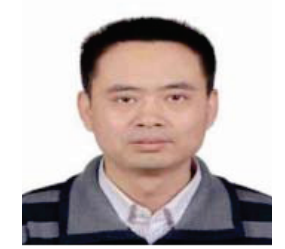

Haipeng Peng received the M.S. degree in system engineering from Shenyang University of Technology, Shenyang, China, in 2006, and the Ph.D. degree in signal and information processing from Beijing University of Posts and Telecommunications, Beijing, China, in 2010. He is currently a Professor with the School of CyberSpace Security, Beijing University of Posts and Telecommunications. He has the coauthored 100 scientific papers. His research interests include information security, network security, complex networks, and control of dynamical 